\title{
Performance of an endcap prototype of the ATLAS Accordion Electromagnetic Calorimeter
}

\section{The RD3 Collaboration}

\author{
D.M.Gingrich ${ }^{1)}$ and J.L.Pinfold \\ University of Alberta, Edmonton, Alberta, Canada \\ E.Boos and B.O.Zhautykov \\ HEPI, Alma-Ata, Kazakhstan
}

B.Aubert, A.Bazan, B.Beaugiraud, J.Boniface, J.Colas, S.Jezequel, T.Leflour, M.Maire, F.Rival, M.Stipčević( ${ }^{2)}$, J.Thion, D.VanDenPlas,

I.Wingerter-Seez, R.Zitoun and Y.P.Zolnierowski

LAPP, Annecy, France

M.Chmeissani, E.Fernandez, Ll.Garrido, M.Martinez and C.Padilla

Univ. Autónoma Barcelona, Bellaterra, Spain

H.A.Gordon, V.Radeka, D.Rahm, and D.Stephani

Brookhaven National Laboratory, Upton, USA

L.Baisin, J.C.Berset, J.L.Chevalley, F.Gianotti ${ }^{3)}$, O.Gildemeister,

C.P.Marin, M.Nessi, L.Poggioli, W.Richter and V.Vuillemin

CERN, Geneva, Switzerland

J.M.Baze, L.Gosset, P.Lavocat, J.P.Lottin, B.Mansoulié, J.P.Meyer, J.F.Renardy, J.Schwindling and J.Teiger

CEA, DSM/DAPNIA/SPP, CE Saclay, Gif-sur-Yvette, France

J.Collot, P.de Saintignon, D.Dzahini, J.Y.Hostachy, G.Laborie,

G.Mahout, E.Merchez and J.Pouxe

ISN, Grenoble, France

L.Hervas L.Labarga, C.V.Scheel

Univ. Autónoma Madrid, Spain

A.Chekhtman, P.Dargent, B.Dinkespiller, F.Etienne,

P.Fassnacht, D.Fouchez, L.Martin, O.Martin, A.Miotto, E.Monnier, E.Nagy, C.Olivetto and S.Tisserant

CPPM Marseille, France

1) Also at TRIUMF, Vancouver, B.C., Canada

2) On leave from Rudjer Bošković Institute, Zagreb, Croatia

3) Also at University of Milano, Italy 
G.Battistoni, D.V.Camin, D.Cavalli, G.Costa, L.Cozzi, S.Resconi,

N. Fedyakin, A.Ferrari, L.Mandelli, M.Mazzanti,

L.Perini and P.Sala

Dipartimento di Fisica dell'Università e Sezione INFN, Milano, Italy

G.Azuelos, G.Beaudoin, P.Depommier, E.León-Florián, C.Leroy and P.Roy University of Montreal, Canada

M.Seman

Nevis Laboratories, Columbia University, Irvington NY, USA

E.Augé, R.Chase, J.C.Chollet, C.de La Taille, L.Fayard, D.Fournier,

A.Hrisoho, B.Merkel, J.M.Noppe, G.Parrour, P.Pétroff, A.Schaffer,

N.Seguin-Moreau, L.Serin, V.Tisserand and I.Vichou

LAL, Orsay, France

B.Canton, J.David, J.F.Genat, D.Imbault, O. Le Dortz,

A.Savoy-Navarro and P.Schwemling

LPNHE, Universites de Paris VI et VII, France

L.O.Eek, B.Lund-Jensen and J.Söderqvist

Royal Institute of Technology, Stockholm, Sweden

M.Lefebvre $^{4)}$, S.Robertson and J.White

University of Victoria, British Columbia, Canada

\section{To be submitted to Nucl. Instr. and Meth.}

4) Also visiting scientist at LAL, Orsay, France 


\section{Abstract}

The design and construction of a lead-liquid argon endcap calorimeter prototype using an accordion geometry and conceived as a sector of the inner wheel of the endcap calorimeter of the future ATLAS experiment at the LHC is described. The performance obtained using electron beam data is presented. The main results are an energy resolution with a sampling term below $11 \% / \sqrt{E(\mathrm{GeV})}$ and a small local constant term, a good linearity of the response with the incident energy and a global constant term of $0.8 \%$ over an extended area in the rapidity range of $2.2<\eta<2.9$. These properties make the design suitable for the ATLAS electromagnetic endcap calorimeter. 


\section{Introduction}

The electromagnetic calorimeter of the future ATLAS [1] [2] experiment at the LHC is designed with a coverage up to a pseudorapidity ${ }^{1)}$ of 3.2. The technology used is liquid argon (LAr) sampling calorimetry with an accordion geometry and a fast readout. This calorimeter is divided into three parts: the barrel, up to a pseudorapidity $\eta=1.4$, and two endcaps, up to $\eta=3.2$. The endcap is designed as two concentric wheels with a separation at $\eta=2.4$.

A large scale prototype of the barrel, conceived as a sector of the ATLAS calorimeter, had been successfully built and tested [3]. The next step was to design and build an endcap prototype, which has been conceived as a sector part of the inner wheel of the ATLAS electromagnetic calorimeter [4]. This prototype has been exposed to high energy electrons at the CERN Super Proton Synchrotron (SPS) to check that its performance fulfills the ATLAS requirements.

The paper is organized as follows. Section 2. describes the mechanical structure and the readout of the calorimeter. The experimental setup for the beam tests is described in section 3., followed in section 4 . by descriptions of the calibration and correction procedures. The main results of the data analysis are presented in section 5., and section 6 . gives the conclusions.

\section{Detector description}

The prototype forms a sector of a flat disk perpendicular to the beam axis and covers the pseudorapidity range from 2.17 to 2.88 , which corresponds to the inner wheel of the 1993 design of the ATLAS endcap electromagnetic calorimeter. The prototype is fully pointing toward a vertex point located at $2.8 \mathrm{~m}$ from the front face. It is $0.546 \mathrm{~m}$ deep with an inner radius of $0.4 \mathrm{~m}$ and an outer radius of $0.96 \mathrm{~m}$. In the following description, the radial direction is perpendicular to the beam direction in LHC configuration.

The prototype consists of a sixth of a full wheel nominally built out of 348 converters and as many readout electrodes. All the plates, converters and electrodes are accordionshaped and arranged radially like wheel spokes with the direction of the waves parallel to the beam axis.

The converters are made of lead plates with a linearly increasing thickness from 1.3 to $2.3 \mathrm{~mm}$ in the radial direction and sandwiched between two $0.2 \mathrm{~mm}$ stainless steel sheets with $0.3 \mathrm{~mm}$ of glue. The electrodes are multilayer copper-kapton boards of thickness 0.3 $\mathrm{mm}$ and are divided into 24 projective strips of constant $\Delta \eta=0.03$. These electrodes are separated from the converters by a LAr gap increasing from 1.95 to $2.65 \mathrm{~mm}$ with increasing radius (fig. 2).

In the azimuthal direction, three strips from consecutive electrode boards are ganged together and thus define cells with a constant $\Delta \phi=0.05$. Each strip has a longitudinal segmentation into three depth regions of $9 X_{0}, 9 X_{0}$ and $6 X_{0}$. The readout cells are defined by $\Delta \eta \times \Delta \phi=0.03 \times 0.05$ for the two first sampling depth regions. For the third depth region, the $\eta$ strips are larger by a factor of two.

The pointing structure in $\phi$ is achieved by opening up the absorber and readout plates around the beam line. The lead sheet thickness and the gap between electrodes and converters increase with the radius as quoted above and the wave angle increases from $57^{\circ}$ to $110^{\circ}$ with the radius. With this geometry, as shown with a detailed Monte

1) The pseudorapidity is defined as $\eta=-\ln \tan (\theta / 2)$ where $\theta$ is the angle with respect to the beam axis. 
Carlo simulation [5], the sampling fraction can be kept nearly constant in both $\eta$ and $\phi$ directions and the amount of LAr traversed almost uniform.

In such a calorimeter with fast shaping of the readout, the signal response depends not only on the total energy deposited in liquid argon but also on the gap width and on the high voltage applied [6].

$$
\text { signal } \propto \frac{X_{\text {lar }}}{X_{\text {tot }}} \cdot \frac{v}{g}=\frac{X_{\text {lar }}}{X_{\text {tot }}} \cdot \frac{(U / g)^{0.3}}{g}=\frac{X_{l a r}}{X_{t o t}} \cdot \frac{U^{0.3}}{g^{1.3}}
$$

where $X_{l a r} / X_{\text {tot }}$ is the sampling fraction, $v$ the drift velocity, $U$ the high voltage and $g$ the gap width.

The response can be kept constant, with constant sampling fraction and radially increasing gap, by radially increasing the high voltage on the electrodes.

In the first approximation, this is achieved by increasing the high voltage in the $\eta$ direction, one value for each pair of adjacent strips, but differing in two longitudinal divisions, on value for the first depth and another for the second and third samplings together. Thus, in our standard operating conditions, twelve different voltages for the first sampling zone and twelve others for the second and third zones were defined. They ranged typically from $1000 \mathrm{~V}$ at low radius to $2500 \mathrm{~V}$ at the largest, according to Monte Carlo studies.

This continuous variation of the detector geometry in the radial direction is a new feature and the main difference with respect to the previous accordion prototypes.

\subsection{Readout electrodes, absorber plates and detector assembly}

The production of the readout electrodes and converters and the assembly of the prototype use similar techniques as those developed for the barrel prototype, already described in ref. [3]. Only the main features and the differences with the barrel prototype are reported here.

The readout electrodes are multilayer boards consisting of two (one double-sided and one single-sided) copper-clad kapton sheets glued together. The copper layers are etched into projective strips separated by $1 \mathrm{~mm}$ gaps for insulation, which define the longitudinal and the $\eta$ segmentation of the detector.

The ionization charge drifting in the LAr gap induces, by capacitive coupling, a current on the inner conductive layer, which is DC coupled to the input of the preamplifier at the beginning of the readout chain. The two outer conductive layers are at high voltage and produce the electric field over the gap.

Each absorber plate consists of a sandwich of five elements: stainless steel, prepreg adhesive, lead, prepreg adhesive, stainless steel. The lead thickness variation is obtained with conventional machining to a precision of $0.02 \mathrm{~mm}$. The lead and stainless steel are first bent, then glued together. The plates were sorted in such a way that the overall rms dispersion of the lead weight from plate to plate was about $0.25 \%$ except on the edge, where it is larger, especially at small $\phi$ values $\left(N_{\phi}=[2-3]\right)$ (fig. 3 ).

The contribution of the lead thickness variation to the signal dispersion can be estimated by multiplying the dispersion of the average of the three lead absorber weights per cell by a factor 0.7 , which corresponds to the signal dependence on the lead thickness [3]. The resulting estimates for the signal dispersion are $0.2 \%$, excluding the two layers $N_{\phi}=[2-3]$, and $0.4 \%$ over the full $\eta$ range of the prototype.

The cylindrical symmetry of the prototype and the correct gap thickness between 
the plates were obtained by gluing the front and the back edges of each absorber to precision wedge-shaped fiberglass bars, which were then piled on top of each other. Honeycomb strips, glued to the converter plates in a few points, were used to center the kapton readout electrodes. Furthermore, in order to cope with the variable gap between kapton and converter plates, these honeycomb strips were machined to have a variable thickness.

The mechanical uniformity was then estimated by measuring the capacitance $C_{g a p}$ of several double gaps consisting of one electrode with an absorber plate on either side. If $2 g$ is the distance between two consecutive converters and $C_{1,2}$ is the capacity of the two single gaps, then [3]

$$
C_{\text {gap }}=C_{1} \| C_{2}=C_{1}+C_{2} \propto \frac{1}{g-\epsilon}+\frac{1}{g+\epsilon} \simeq \frac{2}{g}
$$

where $\epsilon$, the displacement of the electrode from the center of the double gap, is supposed to be negligible with respect to g. The capacitance measurements are presented in fig. 4 . A small relative displacement $\epsilon$ of the gap gives rise to a relative signal deviation of $\epsilon / 2$, as demonstrated in ref. [7] by taking the derivative of equation 1 and taking into account the variation of the sampling fraction with the gap. We measured an rms dispersion of the capacitance values of $2.6 \%$, which thus corresponds to a signal dispersion of $1.3 \%$ on the readout electrodes. After grouping the readout electrodes to form cells, as explained in next section, and grouping cells to form an electromagnetic cluster, this gap nonuniformity corresponds to a dispersion on the signal of an electron shower of $0.4 \%$.

Several reasons may account for these small deformations of the mechanical part. The prototype was dismantled in order to change kapton boards, and indeed the capacitance measurements of the gaps carried out on the prototype before its dismantling showed a much more uniform mechanical structure than afterwards. In addition, mechanical deformations may have occurred due to the rotation of the prototype inside the cryostat during the data-taking period.

Obviously, special care has to be taken in all such operations in order to keep the detector structure sufficiently rigid and uniform.

\section{$2.2 \quad$ Readout electronics}

The signal readout electrodes are grouped together to form cells. This was achieved on the mother boards plugged onto the kapton foils. Care has been taken in their design to have all the signal strips of a mother board isochronous within $0.1 \mathrm{~ns}$. The front-end electronics consist of a warm current preamplifier placed outside the cryostat through a 50 $\Omega$ cable. The preamplifier output was shaped with a $\mathrm{CRRC}^{2}$ unipolar filter. The peaking time $t_{p}(\delta)$ of the shaped response to a short current pulse was $18 \mathrm{~ns}$, corresponding to about $35 \mathrm{~ns}$ for the triangular ionization signal from the detector. The shaper was followed by a track-and-hold $(\mathrm{T} \& \mathrm{H})$ circuit whose timing was adjusted to sample the shaper signal near the maximum. The output was digitized by 12-bit charge integrating ADC.

Due to the increase of the cell capacitance with respect to the radius, which induces a variation of the peaking time, we have grouped the channels by six consecutive values in $\eta$ and four in $\phi$ on the same T\&H.

The coherent noise, usually associated with improper shielding and ground loops, has been measured to be $6 \mathrm{MeV}, 6 \mathrm{MeV}$ and $7 \mathrm{MeV}$ per channel in the first, second and third samplings, respectively. 
The incoherent noise observed in the calorimeter comes mainly from the preamplifier noise and varies with $\eta$ due to the capacitance variation. It has been measured to be between 46 and $52 \mathrm{MeV}$ per channel in the first sampling, between 46 and $56 \mathrm{MeV}$ in the second and third samplings.

The crosstalk between adjacent cells has been estimated using the calibration data and found to be at the level of $1 \%$ between neighbors. Therefore it has a negligible effect on the performance and has not been corrected for.

\subsection{The Monte Carlo program for the prototype simulation}

For the simulation of the endcap prototype performance, a GEANT [8] based Monte Carlo program has been written [5]. It contains a detailed description of the detector geometry where all the parameters of the accordion shape (opening angles, absorbers thicknesses and gaps between the absorbers and the electrodes) depend on the distance from the detector axis.

The simulation takes into account the effects of charge collection, the non-uniformity of the electric field in the fold region and the high voltage variation. It correctly reproduces the division of the prototype into the three samplings and the projective cells in the $\eta$ direction.

\section{Test beam setup}

The data have been taken in the H8 beam line of the CERN SPS using electrons from 20 to $300 \mathrm{GeV}$ during two periods, in November 1993 and in April 1994.

The calorimeter was installed inside a cryostat consisting of a $1.2 \mathrm{~mm}$ thick aluminium outer wall, followed by $30 \mathrm{~cm}$ of low density foam and by an $8 \mathrm{~mm}$ thick stainless steel inner vessel.

In order to have the same incidence angle as a particle originating from the interaction point in collider mode, a simulated interaction point at $2.8 \mathrm{~m}$ was defined. To be able to use the same platform to move in $\eta$ as for the barrel prototype and to compensate for the differences in the rotation lever arms $(2.8 \mathrm{~m}$ instead of $1.3 \mathrm{~m}$ for the barrel), the calorimeter was installed in the back of the cryostat. Rohacell foam was put in front as an argon excluder.

The total amount of passive material in front of the prototype was about $0.9 X_{0}$ at $\eta=2.9$.

The calorimeter could be rotated both in the $\eta$ and $\phi$ directions in order to have the beam hit the selected cells along their projective axis. The cryostat was mounted on a computer controlled platform that allowed the prototype to rotate in $\eta$. The rotation in $\phi$ was achieved with a motor operating in the liquid argon.

The beam line was equipped with three proportional wire chambers, used to determine the direction of the incident particles and their impact point on the calorimeter. In addition, four scintillator counters were used to define the spot size and to provide signals for the trigger.

\section{Calibration and corrections}

A first pass was made to compute the calibration and some precise corrections before extracting the real performance of this calorimeter in terms of precise measurements. The different steps are described in the following subsections. 


\subsection{Calibration and timing}

Each channel is calibrated with the "voltage driven" calibration system described in detail in [3]. A voltage pulse with a risetime of less than $1 \mathrm{~ns}$ and an exponential delay with a time constant of $400 \mathrm{~ns}$ simulating the initial part of the electron drift is injected onto the mother board with a $2 \mathrm{k} \Omega$ resistor.

The calibration signals and the signals from electron showers are timed in such a way that the shaper response from each channel is sampling at its maximum by the T\&H circuit. This is achieved with a two-fold procedure described in detail in [3]. In the first step the strobe to the T\&H circuits was timed, with respect to the peak of the calibration pulse, with delay cables. One T\&H circuit was connected to a group of 24 cells. In each group a small variation of the peaking time, i.e., the delay of the maximum of the shaper signal, was measured along the $\eta$ direction. This variation is due to the variation of the cell capacitances. In the second step the strobe to the $\mathrm{T} \& \mathrm{H}$ circuits was timed, with a programmable delay, with respect to the trigger of the incident particles to maximize the calorimeter response. After tuning the delays and unfolding the $\eta$ dependence, the resulting distribution of peaking times has a dispersion of $0.44 \mathrm{~ns}$.

Once the electronic chain was correctly timed, calibration coefficients were determined by fitting the ADC response of each channel to a set of test pulse values with a third order polynomial. With this calibration system, a channel-to-channel non-uniformity of $0.35 \%$ has been measured [3].

\subsection{High Voltage adjustment}

The high voltage should be increased radially, i.e., approximately along the $\eta$ direction, to keep the response of the calorimeter constant (section 2.). A set of theoretical high voltage values obtained from the Monte Carlo were tuned slightly after a first scan and then used during both data-taking periods.

Since the high voltage values are distributed by each pair of adjacent $\eta$ cells, a fine radial dependence across each pair is expected. Such a feature clearly appears in the data: fig. 5 shows the energy response of each cell after normalization to the mean response of each pair of cells at the same HV.

This effect is in agreement with the behavior observed in the MC data and is corrected offline with a linear function each for the even and odd sets of cells.

\subsection{Response variation across a cell}

Non-uniformity of the calorimeter signal with position can deteriorate the energy resolution greatly, and therefore should be minimized. Whereas mechanical nonuniformities can be reduced with careful construction but cannot be removed later, periodic variation of the signal over a cell due to geometrical properties can be corrected for. The corrections are expected to vary with $\eta$ along with the geometry parameters.

The variation of the calorimeter response over a cell to electrons in the $\eta$ and $\phi$ directions is shown in fig. 6 at four positions in $\eta$. This response is reconstructed by summing the energy contained in a $5(\eta) \times 3(\phi)$ cluster of cells around the cell with the maximum energy. The position is computed from the energy barycenter of the electromagnetic shower (section 5.).

In the $\eta$ direction, the parabolic shape reflects the separation between two cells and the lateral leakage from the cluster, as shown in fig. 7. Also evident in fig. 7 is an overall slope of the signal from cell to cell, as a result of increasing cell size, and hence 
containment, with radius. This effect can be corrected by a linear function, and then the same second-order polynomial can be used to correct for the variation across each cell.

In the $\phi$ direction, the signal modulation corresponds to the residual variations of the accordion shape. The Monte Carlo simulation shows that the calorimeter response as a function of $\phi$ has periodic modulations due to two effects: non-uniformity of the sampling fraction near the absorber fold and non-uniformity of the electric field between the absorber and electrode folds. The sum of these effects produces two sets of peaks with different amplitudes and widths at the positions of the absorber and the electrode folds, as shown in fig. 8. This variation can be parametrized and corrected with the sum of a second order polynomial function and Lorentz functions, with a small radial dependence (fig. 6).

The signal modulation with $\phi$ is well reproduced in the Monte Carlo data when the opening angles of the waves are slightly adjusted from the nominal values. This slight modification is expected to come from a change in the accordion shape at liquid argon temperature, but this has not been checked with a full calculation [5]. In addition, this shows that the $\phi$ modulation is sensitive to small mechanical deformations of the absorbers.

\subsection{Longitudinal leakage}

As the total calorimeter depth is only $24 X_{0}$, the longitudinal leakage is not negligible in terms of energy resolution at high energy.

The leakage energy can be recovered by exploiting its linear relation with the energy $E_{s 3}$ deposited in the third sampling. From Monte Carlo simulation, the leakage energy was found to vary as 0.3. $E_{s 3}$. The total amount of energy of the incident particle $E_{\text {tot }}$ can then be found by using the following parametrization: $E_{t o t}=E_{\text {calo }}+0.3 . E_{s 3}$, where $E_{\text {calo }}$ is the energy deposited in the calorimeter. This formula is used in the following analysis. After applying this correction to the $287.5 \mathrm{GeV}$ test beam data, the energy resolution improved by $0.2 \%$.

\section{Detector performance results}

An electron shower is reconstructed by summing the cell responses of a $5(\eta) \times 3$ $(\phi)$ matrix for the first two samplings and of a $3(\eta) \times 3(\phi)$ matrix for the third sampling (where the granularity is twice coarser in $\eta$ ). This corresponds to a front calorimeter window of about $6 \times 6 \mathrm{~cm}^{2}\left(12 \times 12 \mathrm{~cm}^{2}\right)$ and an energy containment of the order of $92 \%(95 \%)$ at the lowest (highest) radius.

\subsection{Energy resolution}

The electron cell position corrections are applied as well as the longitudinal correction, as described in section 4 .. The signal was also corrected for a small energy loss by showers that develop early due to material in front of the prototype [9].

The energy resolution as a function of the beam energy has been measured for four different $\eta$ values (fig. 9). The contribution due to the beam momentum spread has been removed. At a given $\eta$ value, the energy resolution values were parametrized with the following quadratic sum.

$$
\frac{\sigma_{E}}{E}=\frac{a}{\sqrt{E}} \oplus \frac{b}{E} \oplus c
$$


where the three terms are the sampling, the noise and the local constant terms, respectively, and $E$ is expressed in $\mathrm{GeV}$. The values of the three coefficients, for the four different $\eta$ values, are given in table 1.

\begin{tabular}{|c|c|c|c|}
\hline$\eta$ [Cell number] & $\begin{array}{c}a: \text { Sampling term } \\
\left(\% \mathrm{GeV}^{-\frac{1}{2}}\right)\end{array}$ & $\begin{array}{c}b: \text { Noise term } \\
(\mathrm{GeV})\end{array}$ & $\begin{array}{c}c: \text { Constant term } \\
(\%)\end{array}$ \\
\hline $2.66[7]$ & $10.2( \pm .3)$ & $0.525( \pm 0.018)$ & $.31( \pm .04)$ \\
\hline $2.57[10]$ & $10.7( \pm .3)$ & $0.550( \pm 0.021)$ & $.30( \pm .04)$ \\
\hline $2.43[15]$ & $10.2( \pm .2)$ & $0.590( \pm 0.015)$ & $.36( \pm .03)$ \\
\hline $2.22[22]$ & $11.2( \pm .3)$ & $0.516( \pm 0.018)$ & $.51( \pm .03)$ \\
\hline
\end{tabular}

Table 1: Energy resolution fit parameters for the four various $\eta$ values.

The energy resolution obtained with the detailed Monte Carlo simulation of the prototype [5], after correction for the $\phi$ modulations and the back leakage, gives a sampling term of $10 \%$ and a constant term of $0.25 \%$.

The sampling term $(a)$ is of the order of $10-11 \%$, in good agreement with the simulation. The noise term $(b)$ has been checked using random triggers and was found to be larger than the electronic noise by about 70-100 MeV. After investigation with measurements of a preshower put in front of the prototype, this excess has been attributed to the degradation of the energy resolution at small energy due to the material in front of the prototype. The constant term $(c)$ reflects mainly the residual modulations in both $\phi$ and $\eta$ directions.

If we exclude the point at $N_{\eta}=22$ (where an additional mechanical non-uniformity is suspected [9]), the $\eta$ dependence of the energy resolution is not significant in either the sampling term or the constant term. The noise term depends slightly on $\eta$ due to the increase of the capacitance.

\section{$5.2 \quad$ Linearity}

The linearity of the signal with energy has been studied with the same data and the same corrections used to determine the energy resolution. The pedestal value, extracted from the events triggered randomly, is subtracted from the mean value of the fitted calorimeter response. The resulting value is then divided by the nominal beam energy and normalized at $100 \mathrm{GeV}$. The non-linearity is well within $\pm 1.0 \%$ in the range 20 to $300 \mathrm{GeV}$ at the four selected $\eta$ values. (see fig. 10).

\subsection{Position resolution}

The shower position, in both $\eta$ and $\phi$ directions, is determined by the energyweighted barycenter of a $3 \times 3$ cell cluster. The resolution in position is obtained by the difference between the shower position calculated in the first sampling and the impact point reconstructed using the three beam chambers.

An $S$ shape correlation typical of pad detectors is observed between the barycenter position and the impact point in the $\eta$ view and corrected with a tangent function. No correction is needed in the $\phi$ view where the accordion waves induce a better sharing of the shower energy among neighboring cells [10]. After subtracting the contribution of the 
beam chambers, the position resolution measured in the $\eta$ and $\phi$ directions is shown on fig. 11.

The measured values have been fitted by a quadratic sum.

$$
\sigma_{\eta, \phi}=\frac{a}{\sqrt{E}} \oplus \frac{b}{E} \oplus c
$$

where the three terms are the sampling, the noise and the local constant term, respectively, and $E$ is expressed in $\mathrm{GeV}$. The results of the fit are given in table 2.

\begin{tabular}{|c|c|c|c|}
\hline & $a\left(\mathrm{~mm} \mathrm{GeV}^{-1 / 2}\right)$ & $b\left(\mathrm{~mm} \mathrm{GeV}^{-1}\right)$ & $c(\mathrm{~mm})$ \\
\hline$\sigma_{\eta}$ at $\eta=2.66$ & $4.25 \pm .47$ & $28.7 \pm 2.7$ & $.185 \pm .047$ \\
\hline$\sigma_{\phi}$ at $\eta=2.57$ & $4.93 \pm .45$ & $23.7 \pm 2.8$ & $.278 \pm .037$ \\
\hline
\end{tabular}

Table 2: Position resolution fit parameters for both views, $\eta$ and $\phi$.

The sampling term increases linearly with radius due to the increase of the cell size. This term scales as $0.17 \mathrm{~mm}$ per cell along the $\eta$ direction.

The values for the sampling term and the constant term are in good agreement with expectations and comparable with those obtained for the large scale barrel prototype [3]. The somewhat larger noise term found by the fit procedure is, in fact, explained by the scattering of the electrons in the cryostat wall located at $140 \mathrm{~cm}$ from the calorimeter front face (section 3.).

\subsection{Cell-to-cell response uniformity}

Response uniformity from cell to cell has been studied in both the $\eta$ and $\phi$ directions with dedicated sets of data. A scan of $197.5 \mathrm{GeV}$ electrons was made across 16 cells in $\eta$ at three $\phi$ positions. The response variation with the position of the shower was corrected using the functions described in section 4.3. The normalized energy response as a function of $N_{\eta}$ over a total of 48 cells is shown on fig. 12. The resulting global response dispersion of those points is $0.55( \pm .08) \%$.

\subsection{Global energy resolution}

The overall energy resolution using a global electron cell position correction function (section 4.3) is computed for the $197.5 \mathrm{GeV}$ electron data set over the 48 cells mentioned in section 5.4. This encompasses about 100000 events over an area of $16(\eta) \times 3(\phi)$ cells. The energy distribution, shown on fig. 13, has a relative dispersion of $1.14 \%$, taken from a Gaussian fit. After subtracting quadratically the contribution of the sampling term $(0.76 \%)$ and noise term $(0.26 \%)$ measured in section 5.1 , we obtain a global constant term of $0.79( \pm .04) \%$. This global constant term is higher than the quadratic sum $(0.65 \%)$ of the cell-to-cell dispersion (0.55\%), illustrated in fig. 12, and the local constant term (0.35\%) obtained from the energy resolution fit (section 5.1). This difference can be attributed to an underestimation of the local constant term due to the use of a local correction for the $\phi$ modulation in the energy resolution determination. The variation from cell to cell of the $\phi$-modulation found in the data can be related to mechanical deformations, as discussed in section 4.3 .

The various sources of instrumental non-uniformity estimated in sections 2. and 4 . are summarized in table 3 . 


\begin{tabular}{|c|c|}
\hline Effect & contribution (\%) \\
\hline Electronics & \\
\hline Calibration & 0.35 \\
\hline Mechanics & \\
\hline residual $\Phi$-modulation & 0.35 \\
gap non-uniformity & 0.4 \\
absorber thickness & $0.2(0.4)$ \\
\hline total $(\oplus)$ & $0.67(0.75)$ \\
\hline
\end{tabular}

Table 3: The different contributions to the constant term. Numbers in parenthesis have been obtained including the $N_{\phi}=[2-3]$ layers with thick absorbers.

Comparing the expected constant term of $0.67 \%$ from table 3 and the constant term of $0.79( \pm .04) \%$ extracted from the scan data, we can conclude that a large part of the constant term can be attributed to known instrumental effects.

\section{Conclusions}

The measured performance of the endcap prototype is very close to that obtained for the large scale barrel prototype in terms of resolution and linearity. This confirms that one can successfully adapt the accordion geometry to an endcap configuration. The constant term over a large area has been found to be somewhat larger. The sources of non-uniformities can be explained mainly by mechanical effects, such as spread in the gap width and lead thickness, and by precision in the calibration. Those effects will be improved in the final large scale construction. This performance makes the design suitable for the ATLAS electromagnetic endcap calorimeter.

\section{Acknowledgements}

We wish to thank the technical and support staffs of the collaborating institutes for their substantial contribution.

\section{REFERENCES}

[1] ATLAS Collaboration, Letter of Intent, CERN/LHCC/92-4 (1992).

[2] ATLAS Collaboration, Technical Proposal, CERN/LHCC/94-43 (1994).

[3] D.M.Gingrich et al., Performance of a Large Scale Prototype of the ATLAS Accordion Electromagnetic Calorimeter, Nucl. Instr. and Meth. A364 (1995) 290.

[4] B.Aubert et al., Status Report and Further R\&D for EM and Hadronic Calorimetry, CERN/DRDC 93-04.

[5] A.Chekhtman, The endcap accordion calorimeter prototype simulation, ATLAS Internal note in preparation.

[6] A. Chekhtman, D. Fouchez, E. Monnier, The accordion in the end-cap: geometry and caracteristics, ATLAS Internal note LARG-NO-04 (1994).

[7] C. de la Taille, L. Serin, Temperature dependence of the ATLAS electromagnetic calorimeter signal. Preliminary drift time measurement. ATLAS Internal note LARGNO-29 (1995).

[8] GEANT Detector Description and Simulation Tool, CERN Program Library Q123. 
[9] A. Chekhtman et al., Performance of a Liquid Argon Electromagnetic Endcap Calorimeter Using an Accordion Geometry, ATLAS Internal note CAL-NO-067 (1995).

[10] B.Aubert et al., Performance of a liquid argon electromagnetic calorimeter with an accordion geometry, Nucl. Instr. and Meth. A309 (1991) 438. 


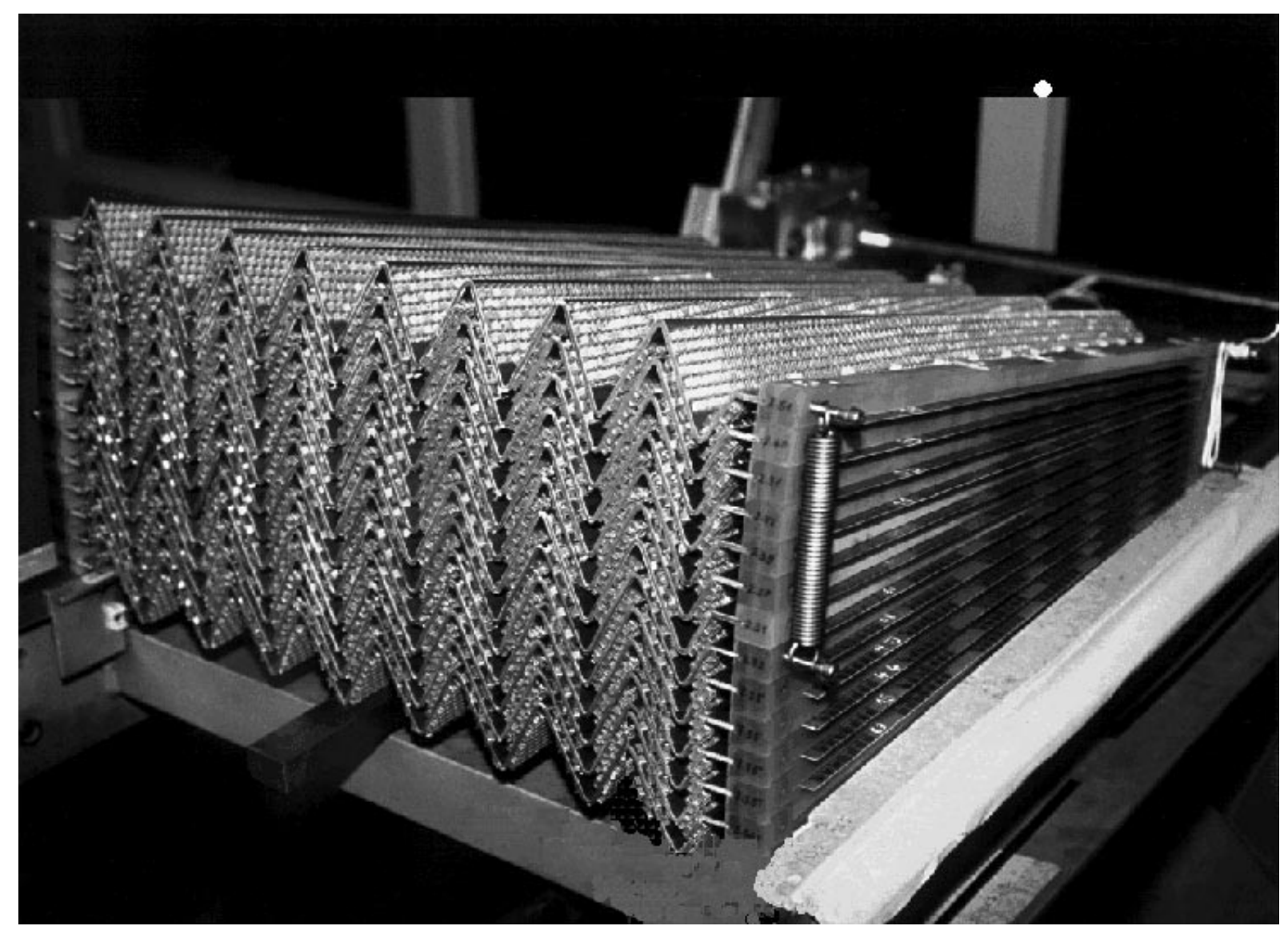

Figure 1: Picture of the prototype taken during assembly. 


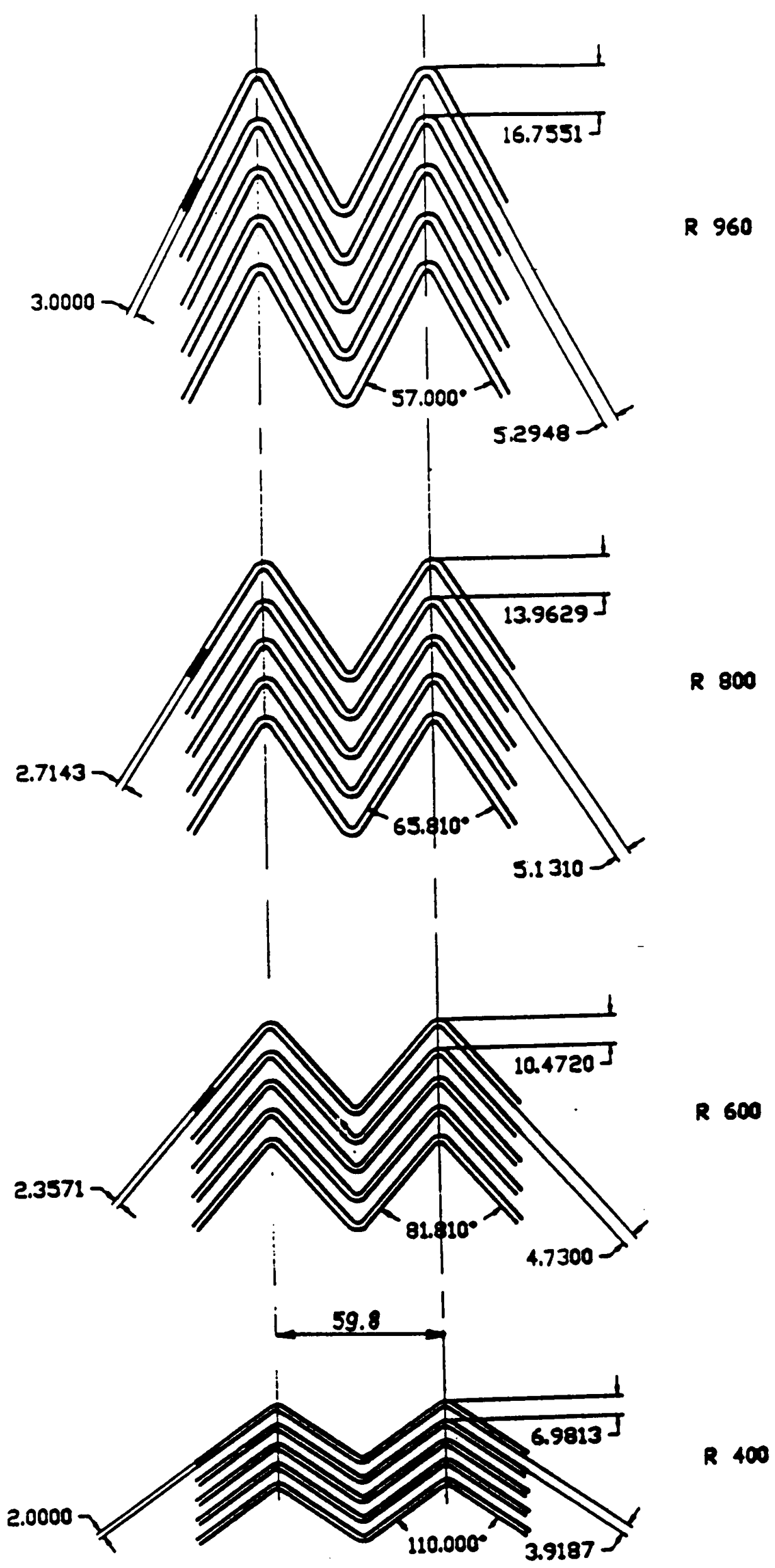

Figure 2: Converter plate profile at four values of the radius. 


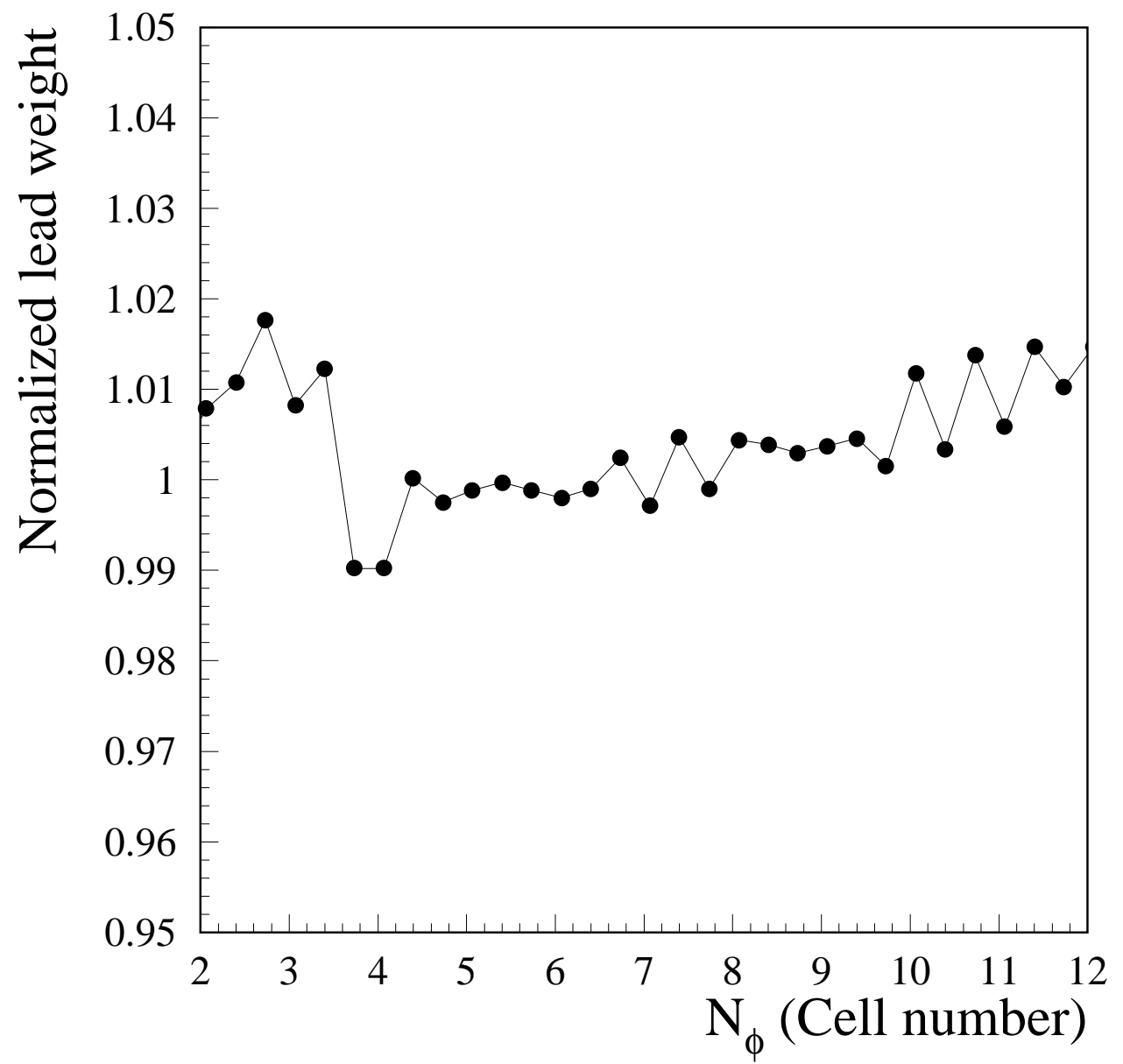

Figure 3: Lead weight variation of each absorber plate versus cell number in the $\phi$ direction. 


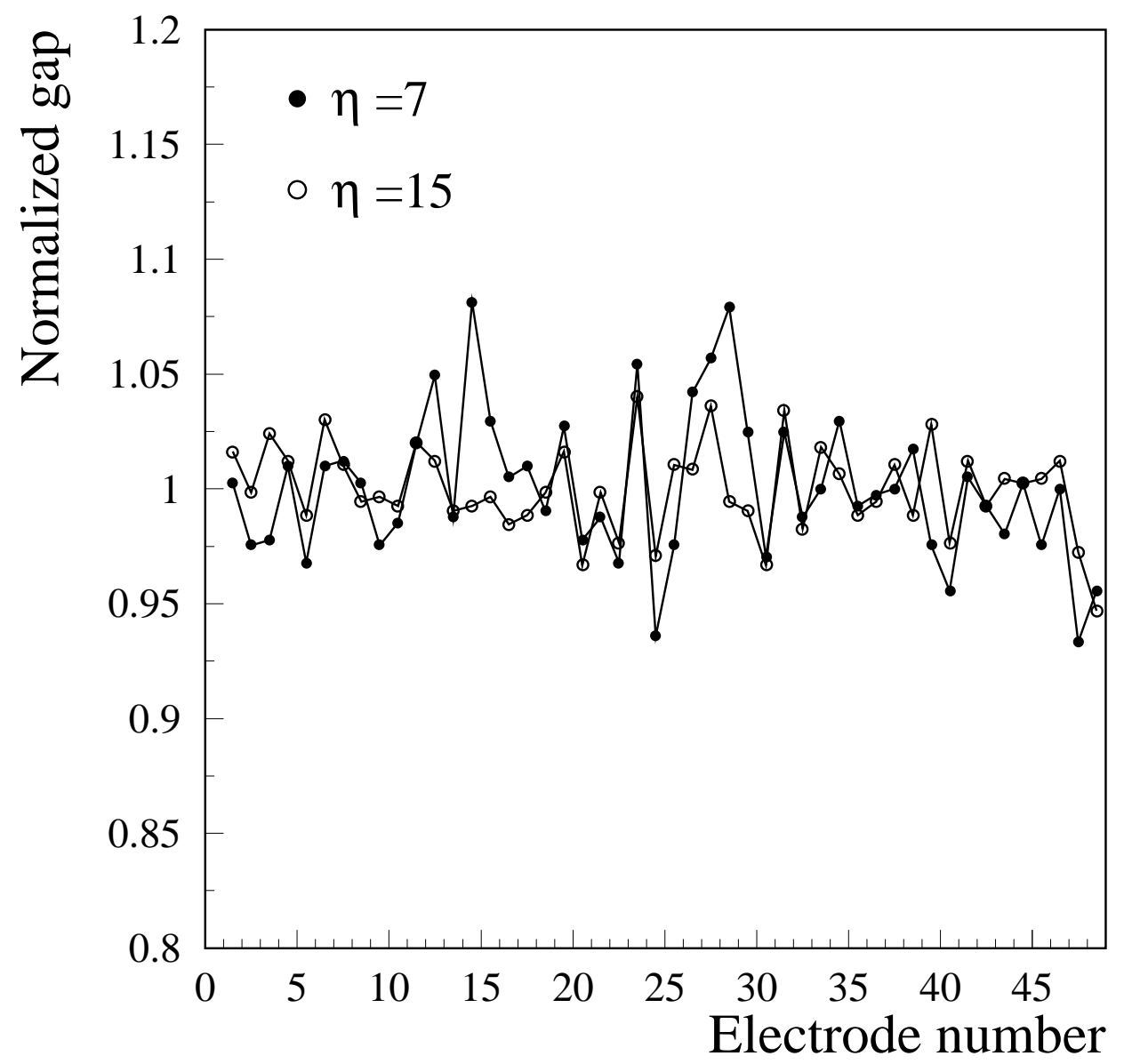

Figure 4: Normalized capacitance as a function of the electrode number along two rows in $\eta$. The normalization is done with the mean value of each $\eta$ row. 


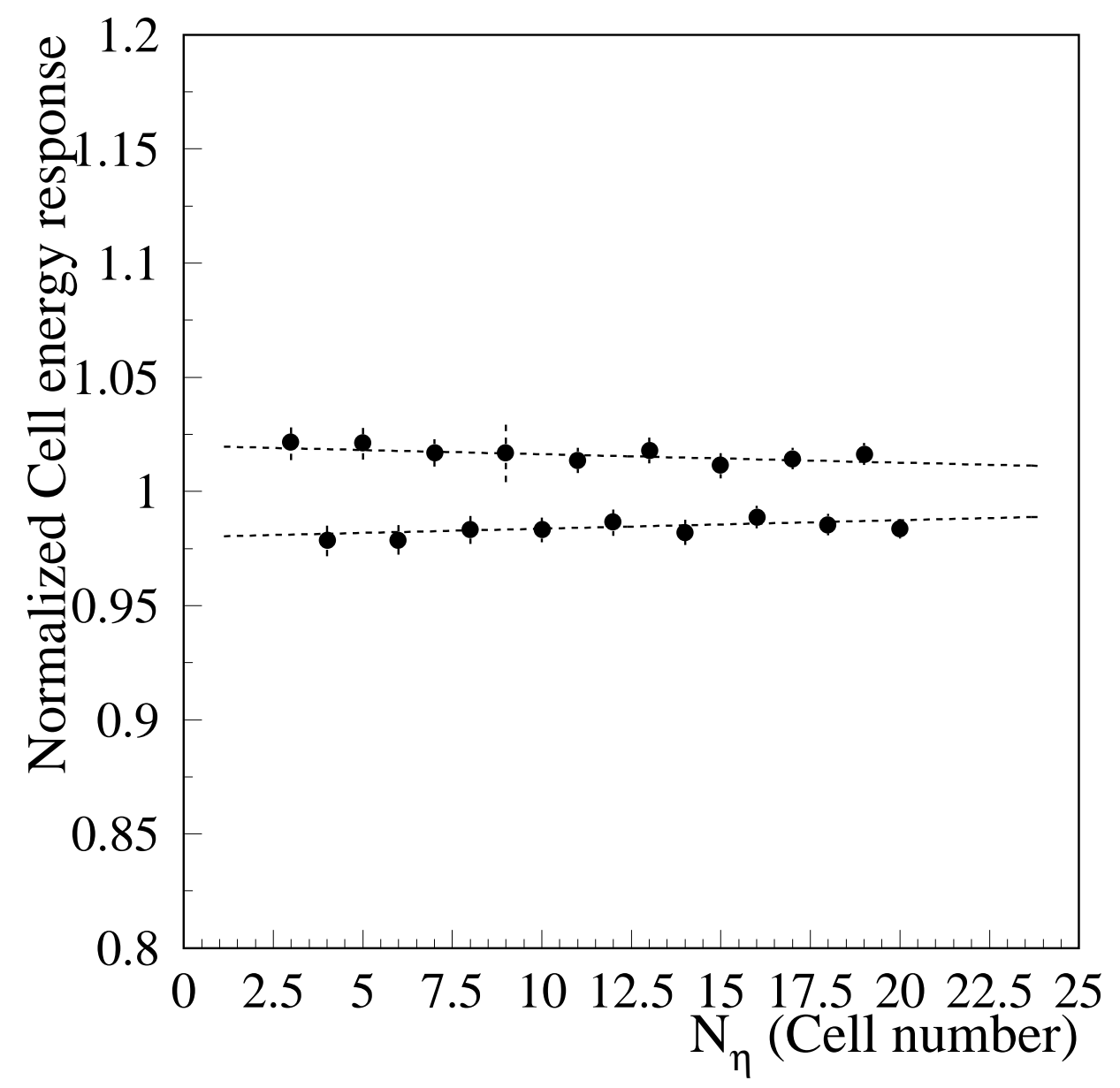

Figure 5: Normalized response of a cell as a function of the cell number in the $\eta$ direction. The dotted lines correspond to linear fits to the odd and even cell response, used to correct an effect of the $H V$ distribution. 

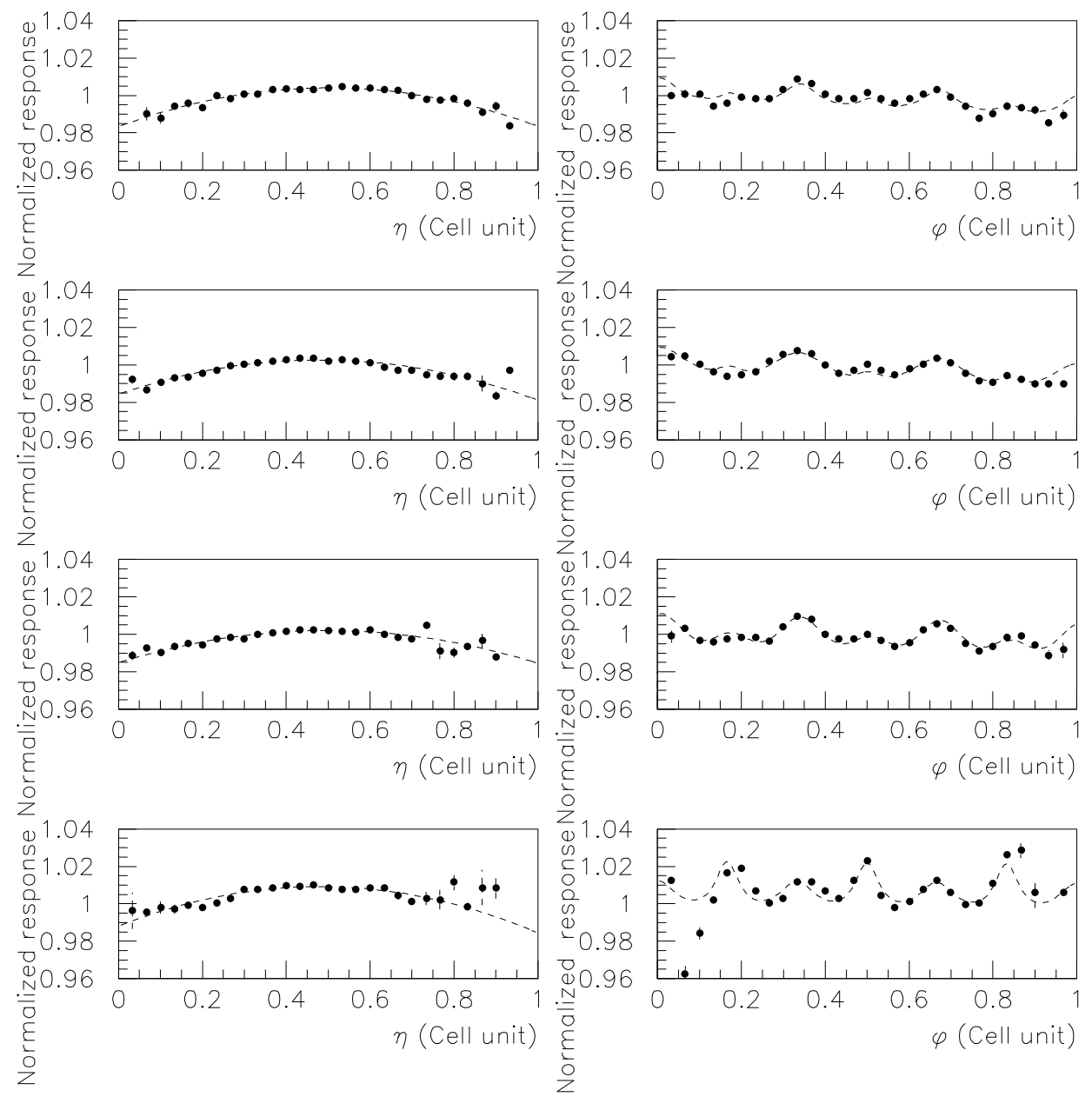

Figure 6: Normalized energy response versus $\eta$ (left) and $\phi$ (right) across a cell for 4 different cells: $N_{\eta}=7,10,15$ and 22 (from top to bottom) 


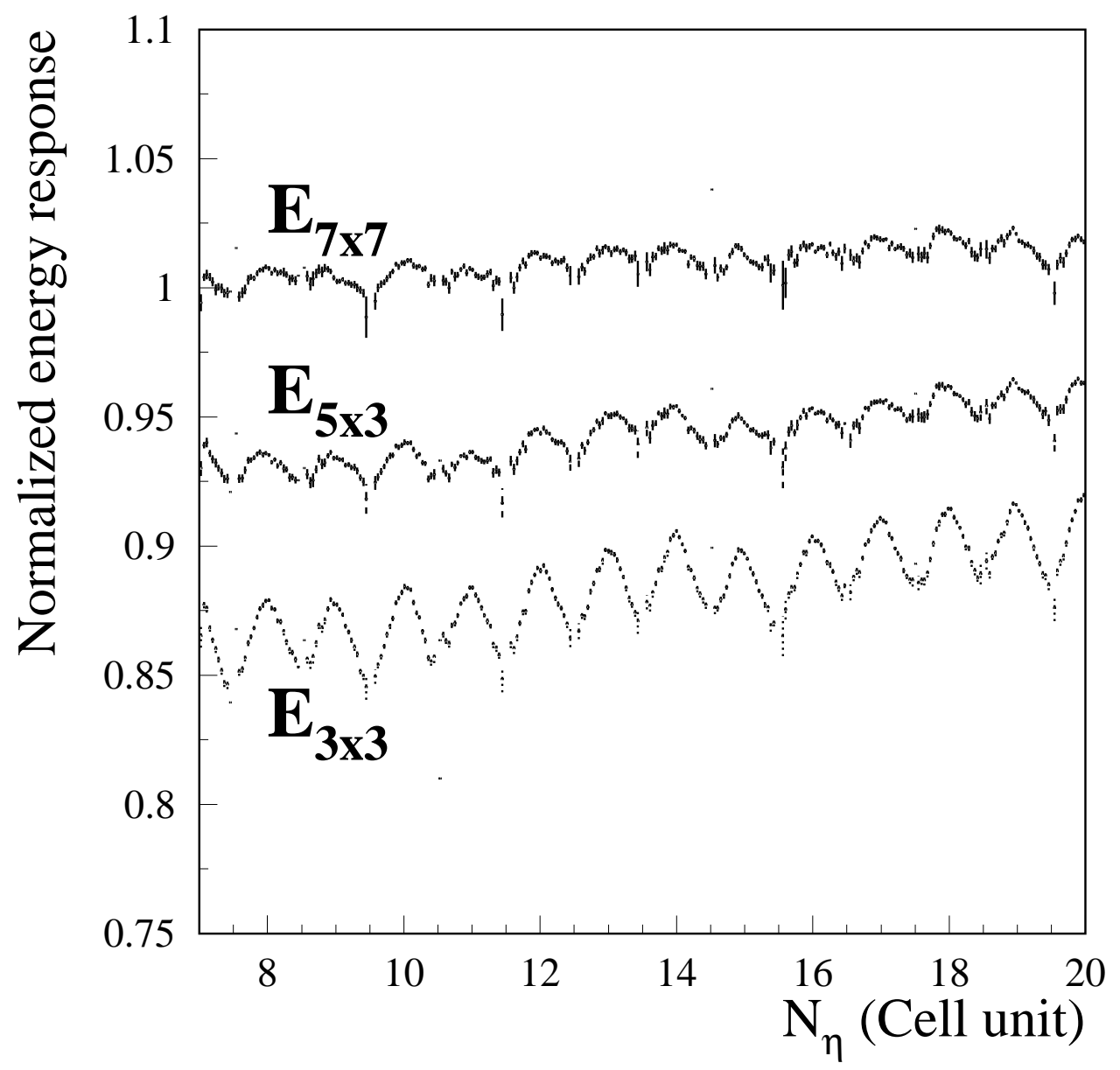

Figure 7: Reconstructed energy response, normalized to the energy in a cluster $7 \times 7$ at $N_{\eta}=7$, versus $\eta$ for three different cluster sizes: $(\eta \times \phi)=3 \times 3,5 \times 3,7 \times 7$. 


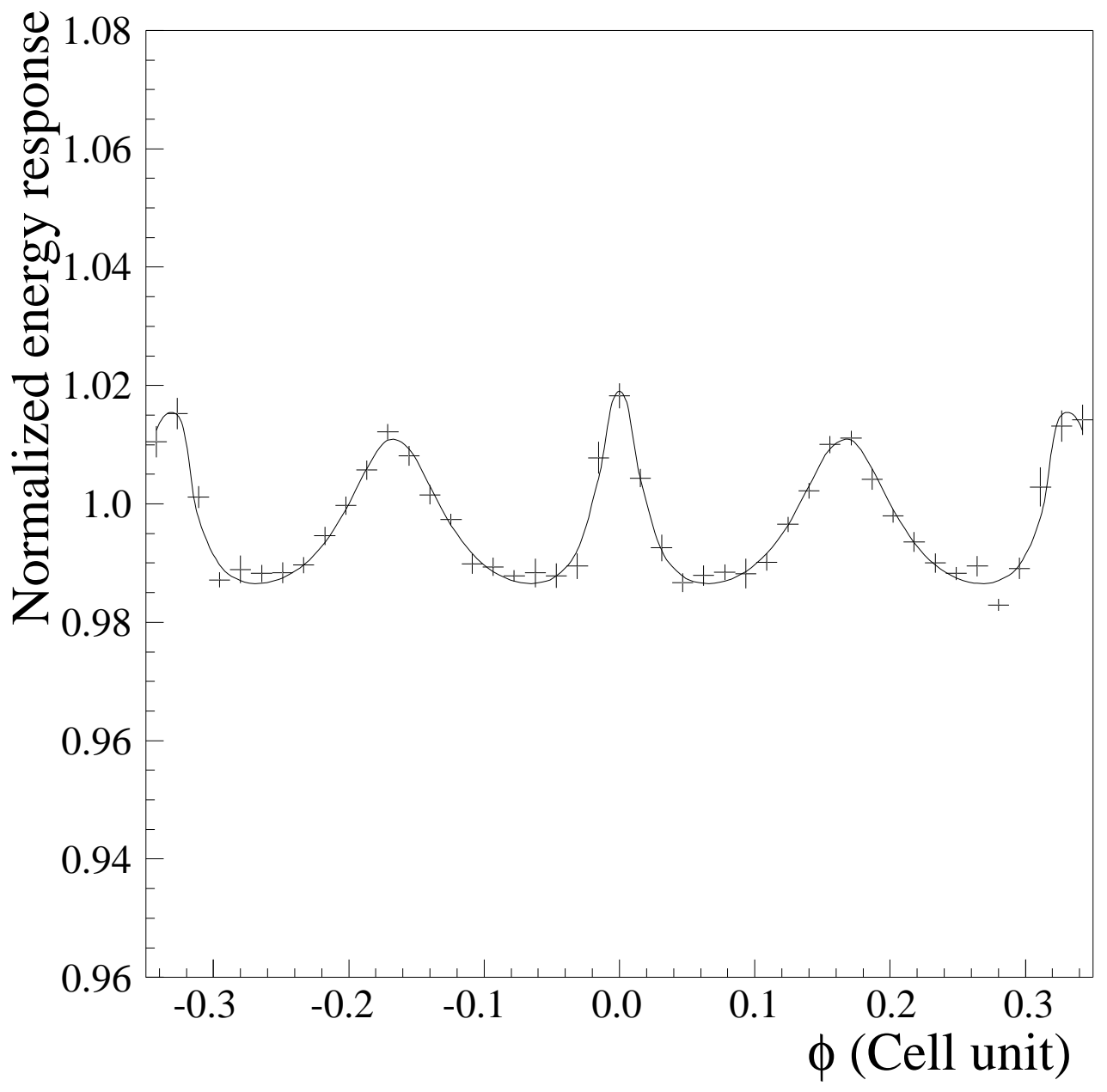

Figure 8: Modulation of the signal along the $\phi$ direction at $N_{\eta}=22$, as obtained by Monte Carlo simulation. The curve is a parametrization of this data. 


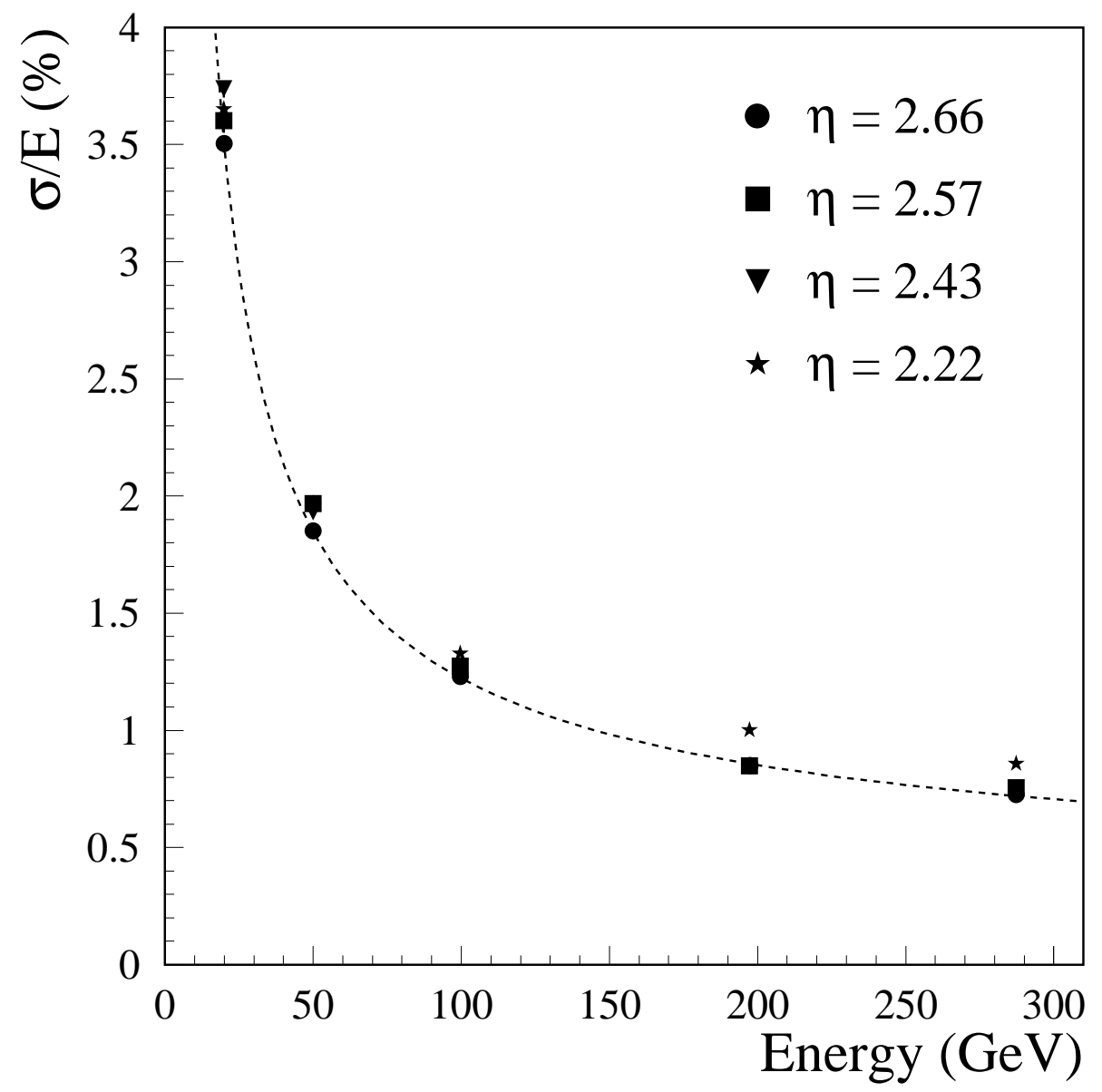

Figure 9: Energy resolution as a function of the incident beam energy at four different $\eta$ values. The dashed line is the best fit to the data at $\eta=2.66$. 


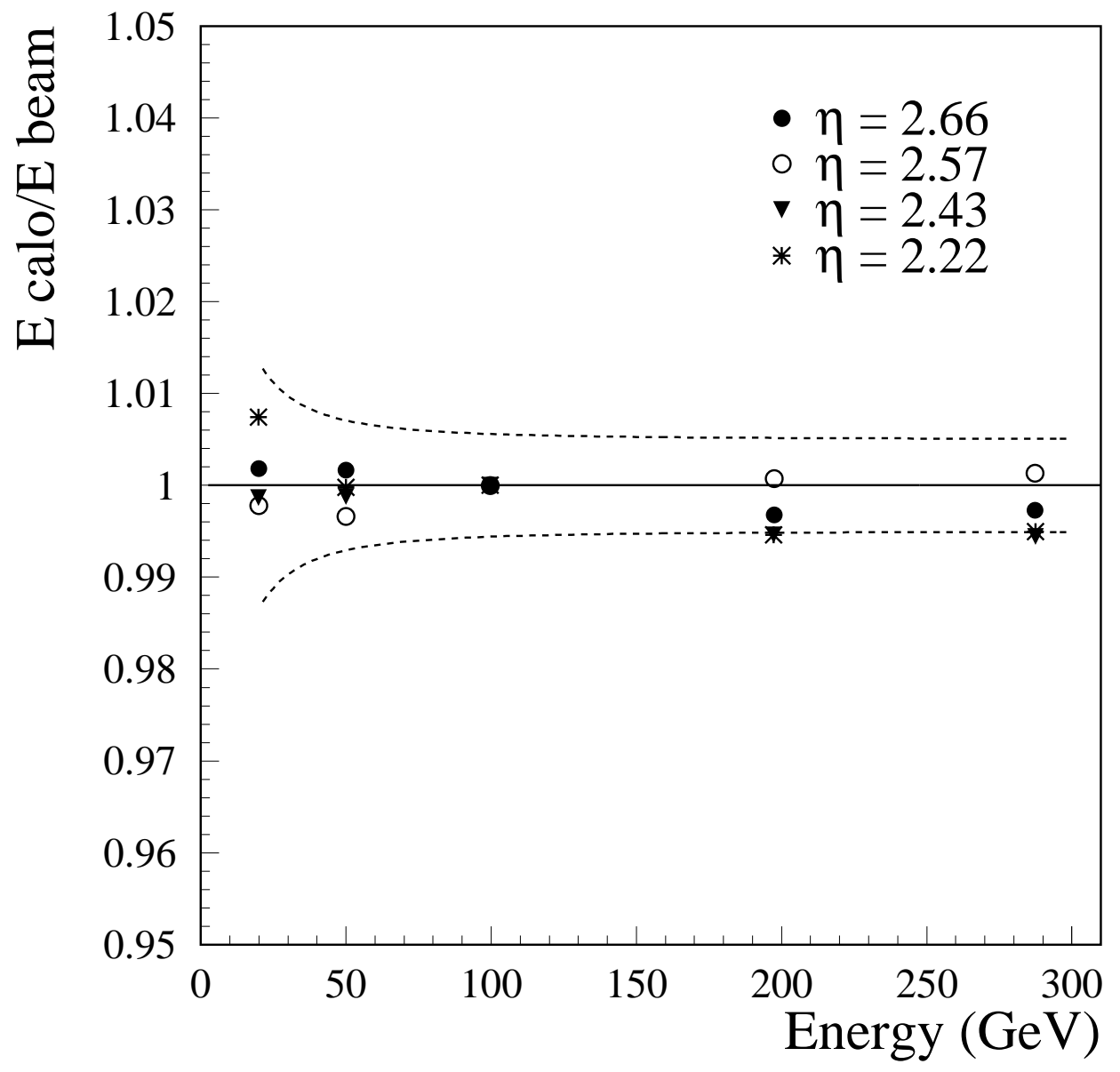

Figure 10: Linearity of the calorimeter response for four cells at different $\eta$ positions. The dashed lines represent the uncertainties in the nominal beam momentum. 

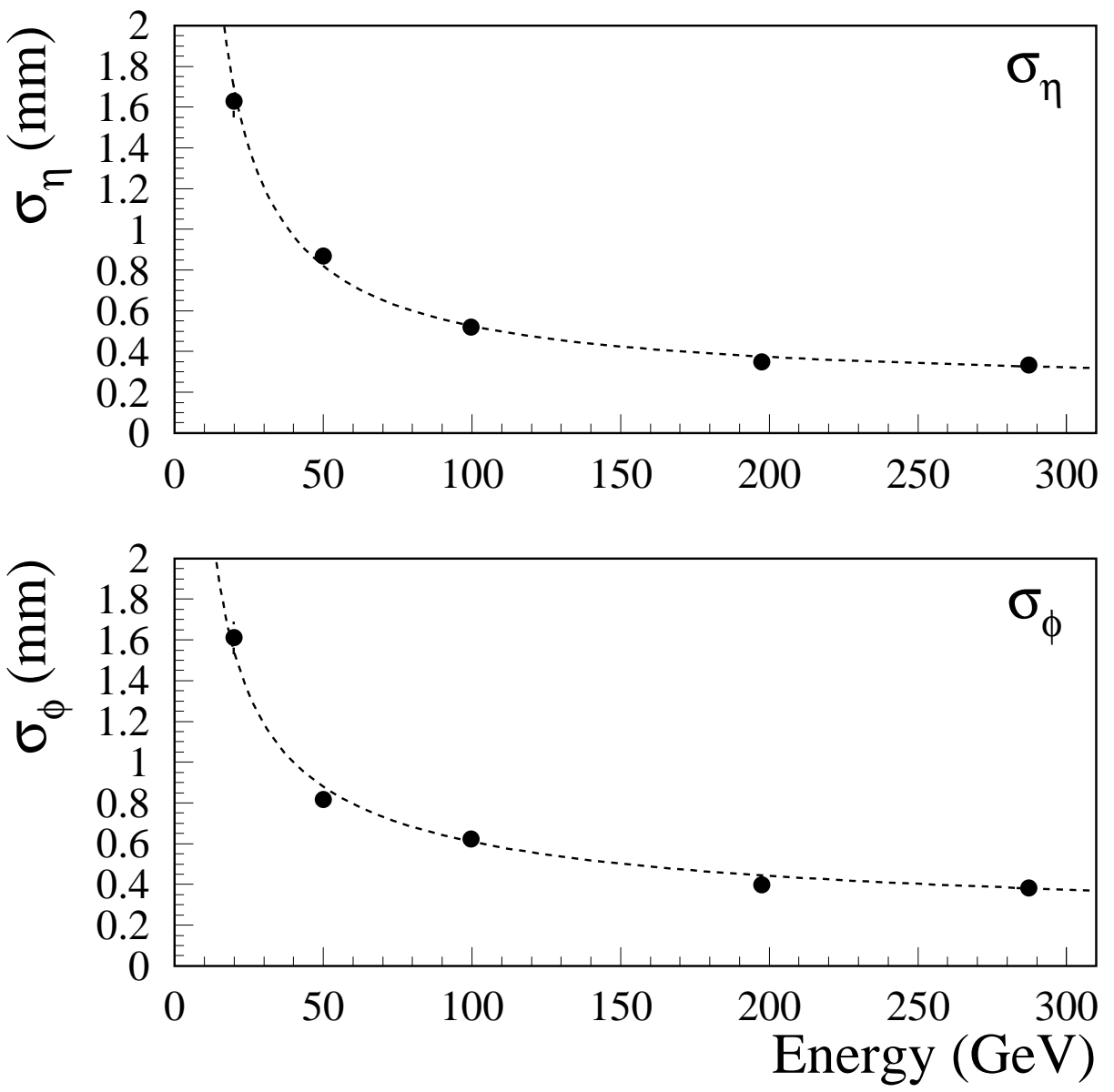

Figure 11: Position resolution in $\eta$ direction at $\eta=2.66$ (top) and position resolution in $\phi$ direction at $\eta=2.57$ (bottom) as a function of energy. The curves are the best fits to the data, according to eq. 4 . 


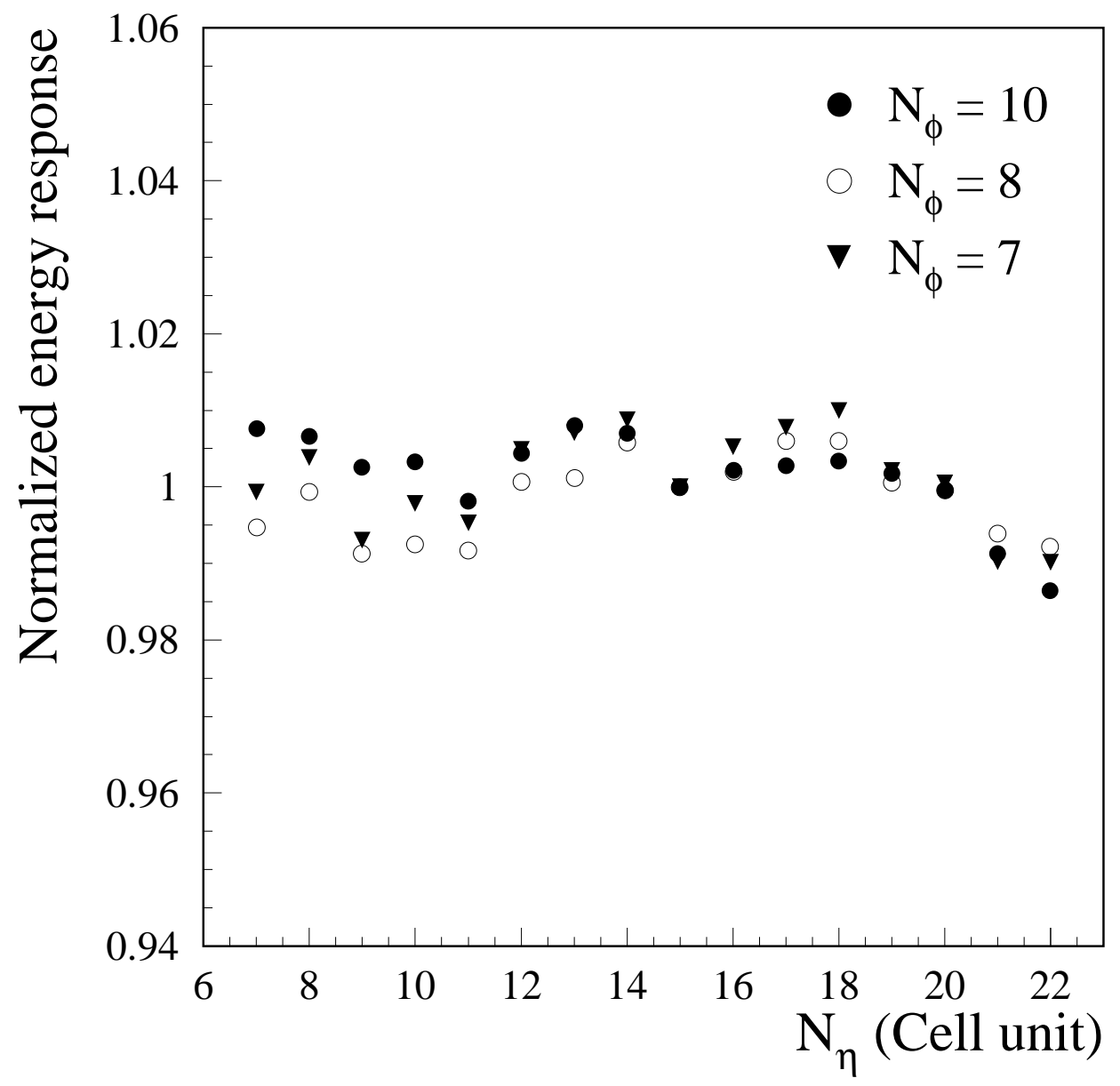

Figure 12: Uniformity along the $\eta$ direction for three different values in $\phi$. 


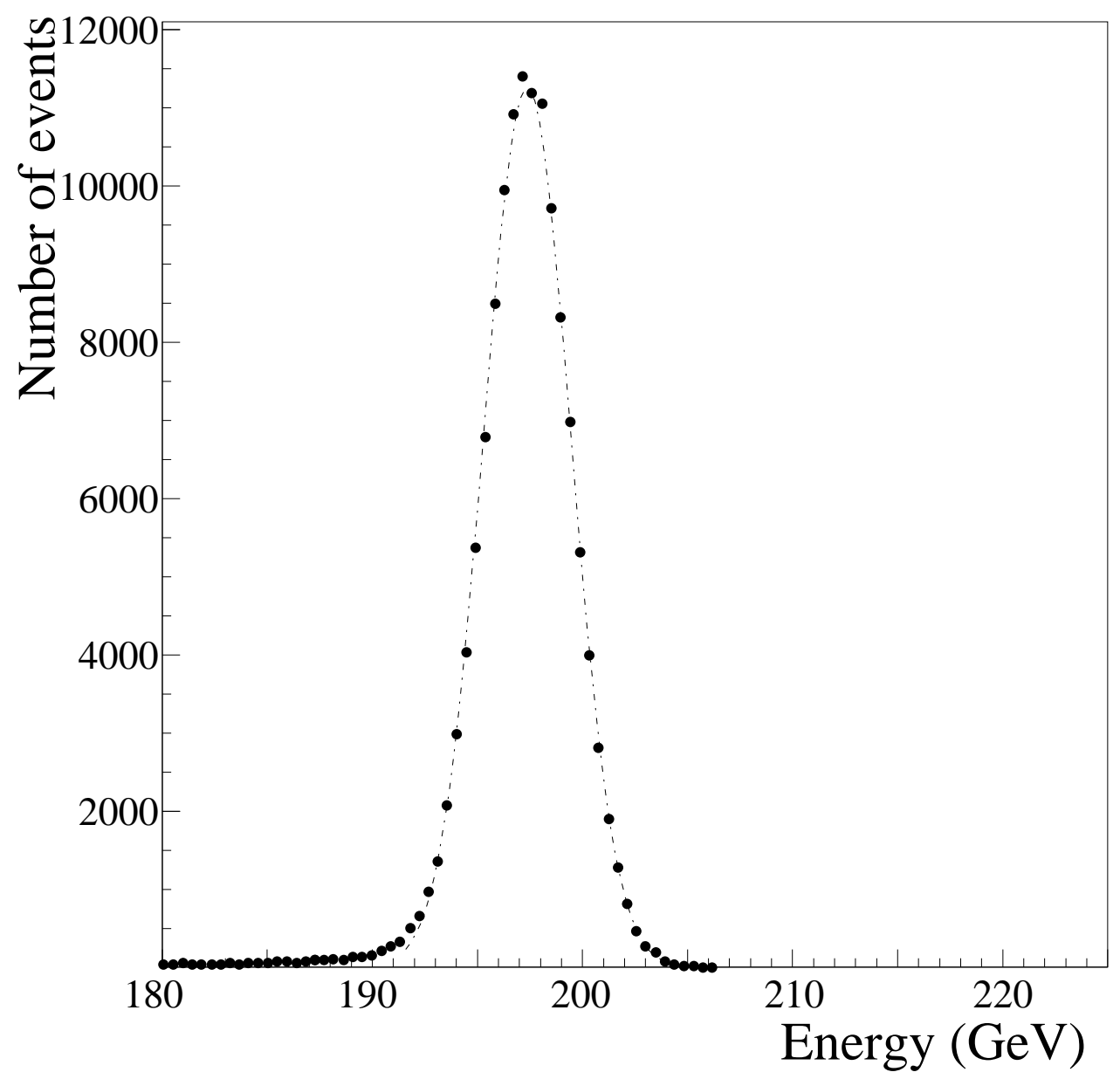

Figure 13: Global energy response to $197.5 \mathrm{GeV}$ electrons over 48 cells. A Gaussian fit, as shown by the dotted line, gives a resolution of $1.14 \%$. 